\title{
Validity and Integrity check in Supply Chain Management using Blockchain
}

\author{
Pranav Gor \\ Dept. of Computer Engineering, \\ DJSCE, Ville Parle, \\ Mumbai, India \\ Deep Gosalia \\ Dept. of Computer Engineering, \\ DJSCE, Ville Parle, \\ Mumbai, India
}

\author{
Rahil Jhaveri \\ Dept. of Computer Engineering, \\ DJSCE, Ville Parle, \\ Mumbai, India \\ Mrs. Aruna Gawade \\ Asst. Prof. \& Assoc. Head, Dept. of Computer \\ Engineering, DJSCE, Ville Parle, \\ Mumbai, India
}

\begin{abstract}
Existing centralized blood supply chain management systems have various drawbacks including lack of information on blood bags, inability to reflect real-time updates in detail and the factor of distrust. Apart from this, our literature survey indicates that the wastage of blood bags is at an all-time high, accredited to the reasons for poor management.

We propose a blood collection chain system based on blockchain technology using a distributed ledger structure and transaction execution of the entire process from blood collection till the blood reaches the final stage - hospitals. In this respect, the blockchain technologies offer the possibility to maintain a transparent blood management system, especially since data cannot be counterfeited and tampered with. In addition, the system stores blood contracts between hospitals in the event of emergencies through the transaction and consensus flows. It will also act as a waste management strategy for Blood Bags by splitting and using various contents in the blood such as RBCs, WBCs, Platelets, Plasma to a maximum extent before its expiry.
\end{abstract}

Keywords-Blood Chain, Blood Traceability, Supply Chain Management, Distributed Ledger, Blockchain, Smart Contracts.

\section{INTRODUCTION}

Every product that we purchase, is in turn connected to a chain of other products that are used as a basic set of ingredients in order to build this very product. In order to ensure that the quality of the end product is up to the required mark, it depends on every single entity in the supply chain to be in place, in a timely manner whilst possessing good quality. It thus goes on to depict that proper management is required to handle the entire process, which is termed as Supply Chain Management (SCM).

Using traditional SCMs, when a product is launched into the market, the consumers are generally skeptical and may have some reservations with respect to the quality of the product. This distrust factor is of primal importance when it is faced in the field of Blood donation and hence has to be rectified as early as possible. Thus, there is a need for a system where a donor can satisfactorily get the whereabouts of the blood he donated and, on the corollary, the recipient of the blood can also be assured that the blood that he is about to be injected with, is $100 \%$ free of any detrimental factors.
According to an article by the Times of India [9], there is a tremendous amount of blood being tagged as 'waste', approximating to nearly six lakh liters of blood over five years of span, in Maharashtra itself and is disposed of due to 'improper coordination between the blood banks and hospitals' with given justification that they expired prior to their use. This shows a lack of management skills against the collection and disposition of the blood bags and is in desperate need of proper maintenance formulation.

In this project, using the Blockchain (BC) implementation, we aim at providing rather simplified yet quality-assured support for maintaining and monitoring the data. It takes into consideration the collection process of the Blood from various different sources along with tracking the physical conditions of those blood bags. The use of $\mathrm{BC}$ also helps provide credibility into the network, encouraging trust and security for the donors and receivers. Our system also handles the issue of excessive wastage by designing a prioritized sequence of dispatch for the blood bags that are sorted according to their expiration dates. We use an urgency factor to determine the status of the emergency and appropriately control the cost structure for the request. This helps the system determine which type of blood bag needs to be transported, that is, in case of emergency, the rule for early expiry bag's dispatch would be suspended.

\section{LITERATURE REVIEW}

Kim S \& Kim D proposed a modification of the existing standard models, that minimizes transportation time of blood during emergencies by not only allowing the blood storage centers to distribute blood but also authorizing specific hospitals as blood distribution institutions [1]. The existing blood information management system manages the information just focusing on the situations before it is supplied from blood banks to a number of medical institutions. Despite efforts to track real-time information on the blood boxes or transport vehicles, there is a limitation to how closely each medical institution conducts blood transfusions and disposal. Besides, in the event of an emergency, there is a possibility that placing temporary facilities or designating a new base for blood supply, does not take realistic complex factors into account. They proposed a system wherein an actor in need of blood can 
view the nearest hospitals or blood centers that have the available blood for distribution thus helping the end-users avoid delays during emergencies.

Blockchain is an ideal platform that can solve a number of problems related to centralized SCM's such as provenance tracking, cost reduction and the most important of all, establishing trust. The paper proposed by el Maouchi, Ersoy and Zekeriya [2] justifies the application of BlockChain in SCM and its feasibility. Their work is generalized for a standard SCM where a product travels from the initial actor to the final actor through the entire cycle and at each exchange, a transaction is validated through consensus and authentication which is then added to the blockchain. The paper provides standard architecture and algorithms to be executed when two actors exchange the product.

The paper proposed by Sylim, Liu, Marcelo and Fontelo focuses on solving one of the major problems faced by the existing SCM in the medical field i.e. drug counterfeiting [3]. The supply chain of medicines is tracked using Ethereum and proof-of-stake algorithm for consensus.

In the field of medicine, blockchain contributes to increased safety and reduced costs which is ensured by identifying changes in ownership of drugs between different participants of the chain such as manufacturers, distributors, packers, and end-users.

In another paper, Feng Tian proposed the use of RFID which allows the users to scan the product and validate its supply chain history, along with Blockchain to track the agri-food Supply Chain [4]. Tian supported the idea that a decentralized approach for tracking products could solve the issues in a centralized approach, such as trust, tampering, etc.

Research on the blood cold chain system has been conducted by several approaches. Davis, Geiger, Gutierrez, Heaser and Veeramani proposed an RFID based system which dynamically manages the information related to blood [5]. Quite a few problems faced by the currently existing system for blood information management were pointed out in [5]. For example, the amount of information that can be contained in the bar code is limited, and the information is not reflected in the blood source in real-time, trusting the respective actors on updating legit information on the system, etc.

Jabbarzadeh, Behnam and Stefan proposed a robust network design model for the blood supply chain in the event of a disaster [6]. They took into consideration a set of decision parameters such as the number of blood centers nearby etc. in order to manage blood allocation, blood collection, and other such critical decisions during a disaster.

\section{PROPOSED MODEL}

In order to maintain transparency in the entire system/network, our solution proposes the use of Blockchain. That is, integrating BC in the SCM network where each and every blood bag can be tracked as per their movement through various actors of the system.

\section{A. $\quad$ Proposed General Architecture}

- The inclusion of Blockchain solves the disadvantage of 'distrust' by providing total transparency to its users. The entities involved in the network are not acquainted with one another which may become a potential cause for dissension during the phase of transactions and payments. Our system resolves this problem, with the help of Smart Contracts, a feature of Ethereum BC, that helps take control of the security aspect of these transactions. Only when a certain criterion is satisfied, the Smart Contract is fired, releasing the due payment.

- Secondly, all the data on a blockchain is stored securely through a cryptographic hash function Keccak-256 and the ledger is owned by each node in the network. This makes it very difficult for any intruder to manipulate the ledger data, ensuring protection against any possible mutations or fraudulent manipulation of the data stored insofar, thus establishing a common base of trust and immutability Also by exposing the supply chain ledger publicly, it helps gain the trust of blood donors.

- Our solution also provides a remedial strategy for improper waste management which is one of the factors that deter donors away from donating blood. Using the data uploaded on the BC network, the internal entities can have access to the transparent data and consequently prioritize the process of dispatching the blood bags that are nearing its expiry. The bags which are nearing their expiry are flagged and are placed as ready to be dispatched in a case when an urgent call from a hospital is received.

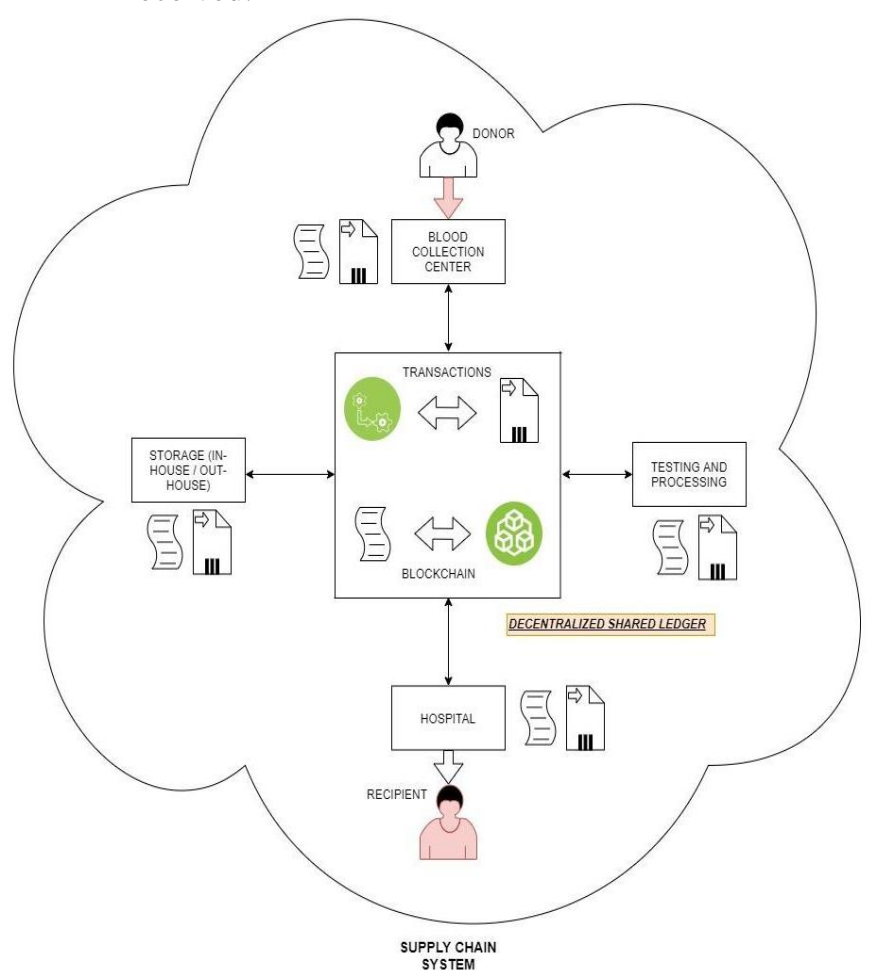

Fig2.1 Proposed Architecture 


\section{B. Certificate}

With respect to the donor, our system will take various parameters as input like the blood group, donation date, expiry date etc. This input obtained will then get hashed for the block creation process and get updated on the BC network. Simultaneously, the Certificate module will get initialized with the corresponding input values. However, the system does not limit itself to the block generation and updating it on the network. Upon further transportation of the blood bags, required entities will generate invoices and update the details regarding the ownership status of the blood bag etc., on to the network as well.

\section{Certificate for BloodMatch}

\begin{tabular}{|c|c|}
\hline *Donor ID (Address) & \\
Donor Blood Group & \\
*Blood_Bag ID (Integer) & *RBC_Bag ID (Integer) \\
Donation_Time (Time) & *WBC_Bag ID (Integer) \\
Blood_Splitted (Bool) & *Plasma_Bag ID (Integer) \\
Location (Latitude, Longitude) & *Platelets_Bag ID (Integer) \\
Expiration (Time) & \\
Owner_name(String) & Expiration_RBC_Bag (Time) \\
Owner ID (Address) & Expiration_WBC_Bag (Time) \\
Availability (Bool) & Expiration_Plasma_Bag (Time) \\
Usage_Status & Expiration_Platelets_Bag (Time) \\
(used/not used/stocked) & \\
\hline
\end{tabular}

Fig2.2 Certificate with required parameters

The working principles:

- The Blood Collection Center (BCC) will create a blood bag with the basic information about it on the Blockchain and send the bag to the Testing and Processing unit (T\&P).

- After testing and processing the blood bag, the system will output the new Unique ID of the split bags (if any). The expiry value for each and every bag received or generated will be updated on to the Database as well as the BC network.

- Next, the blood bag reaches the Storage Center (SCT), where all the physical measures such as temperature conditions, preservation and anticoagulation parameters, are regulated.

- Now as and when the requirement for the blood arises from the hospital, an appropriate urgency request is broadcasted to the nearby SCT and Hospitals, stating the precise values for the requirements. The system then calculates the cost structure and handles the payment of the transaction according to the various urgency-based scenarios. After the transaction is completed the details are updated on the Database and Network.

C. Users of the System

1. The donor will be registered on to the network on donating for the first time and he/she can then login to the application and see/track his donations through a certificate on the web/app UI. The Donor will only be able to view the necessary details about his donated blood on the app.

2. Blood collection centers (BCC) receive donations and for every new blood bag donated, it creates a new blood bag entry (with initial information) and updates it on the $\mathrm{BC}$ which will be the genesis block. BCC can make a transaction of blood bags to the testing and processing center (T\&P).

3. Testing and processing centers (T\&P) accept transactions from BCC and add necessary information about tests performed on the blood and then make a transaction to the Storage center (SCT).

4. Storage center (SCT) receives bags from T\&P thus claiming the ownership of the bag for storing it under proper conditions until a request has been placed for the supply of blood by any hospital. On receiving a request to send blood, SCT then performs a transaction to hand over the ownership of the blood bag to the hospital and on a successful transaction, SCT gets paid for the same through Smart contracts (SCs).

5. Hospitals receive bags from SC and/or other hospitals that also act as suppliers on having excess blood at their disposal, on putting up a request for it. Hospitals can also get requests for blood from nearby hospitals. Finally, hospitals update the information of blood bags depending on whether they were used for any patients or are stored for future use, etc.

\section{Block Transactions}

In the distributed ledger structure, all transactions are recorded and shared within the blood cold chain network. The BCC block collects information about the blood and the transaction is recorded. In T\&P Block, there is a transaction in which the blood that has been judged as conforming moves to the Storage unit. The Storage Block checks parameters for proper storage and indicates that transactions have occurred in which the blood is transferred to the hospital upon request of the hospital. In Hospital Block, the ownership status will be updated. The Boolean parameters viz. Usage Status and Availability will be updated after the requirement of Blood Bag has been satisfied. While processing the smart contract like this, the invoked transactions are recorded like logs.

The Location and Ownership Status will be updated as and when the Blood Bag reaches a particular user (module) of the system. 


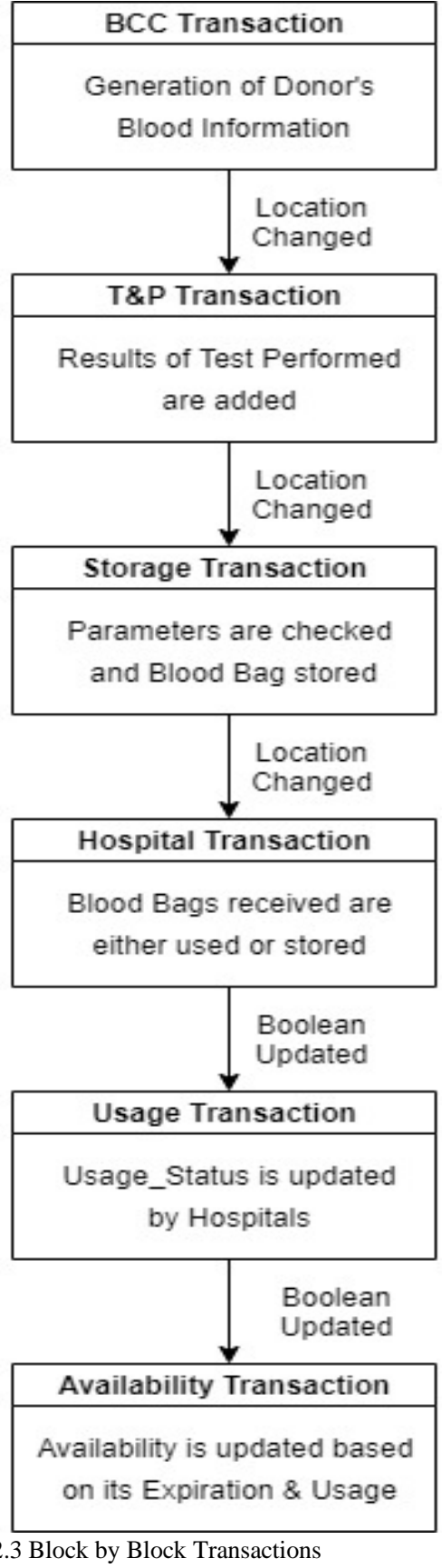

Fig2.3 Block by Block Transactions

\section{CHARACTERISTICS}

Some characteristics of Blockchain-based Blood SCM:

- Openness: The nodes in the blockchain show interoperability that means that the nodes in the Blockchain have the ability to exchange and use information during a transaction.

- Concurrency: Nodes process concurrently to enhance the performance of the blockchain.

- Fault tolerance: The transactions in the Blockchain are immutable and any fault at any node will be transparent to all other nodes in the Blockchain.

- Scalability: Nodes can be added or removed to make the Blockchain flexible. Scalability considers three things: -

1. Size: Mores nodes can be added easily in the Blockchain network.
2. Distribution/Transaction Processing rate: Geographical dispersion of the nodes does not degrade the performance of the Blockchain.

3. Manageability/latency: The Blockchain should be manageable as no. of nodes in the Blockchain increases and the different nodes are located in different parts of the world.

- Transparency: Every transaction in the Blockchain is visible to each node in the blockchain network.

- Security: The Blockchain uses strong Cryptographic protocols such as SHA-256 for securing the data in the Blockchain.

- Quality of Service: The quality of service (QoS) determines the reliability and response time and throughput, that is the system's ability to deliver its services dependably with response time and throughput that is satisfactory to the nodes in the Blockchain. Quality of Service is a critical parameter for critical data transactions among the nodes.

- Efficient Blood Use: Since the quick supply of blood in golden time is an important issue, we make use of a system that enables the supply of blood directly between hospitals. This is when blood is not supplied from the blood banks to the hospitals within the golden time, and the surplus blood is requested from the nearby hospitals.

- Waste Management: The Blood Bag which is near its expiration will be dispatched first when a request is generated.

\section{CONCLUSION}

The existing cold blood chain system lacks reliability, proper waste management system, and immutable transactions. With addition of Blockchain to Supply Chains, it now records the transactions which bolster reliability, maintain $\operatorname{logs}$ and provide traceability. The security of payments and waste management factors have been considered by including smart contracts and deploying near to expiration, blood first processes respectively.

By applying validity and integrity checks at every stage in the Blockchain-based SCM, we have ensured that a scarce and perishable resource like blood is properly collected, stored and dispatched according to their priority for patients. Since the whole process is transparent with the help of the shared ledger maintained, the donors can easily track their donations. It would make a great impact on increasing the trust factor and consequently, the donation rate.

\section{REFERENCES}

[1] Kim S \& Kim D. "Design of an innovative blood cold chain management system using blockchain technologies". In: ICIC Express Letters, Part B: Applications. 9. (2018), pp. 1067-1073.

[2] el Maouchi, Mourad \& Ersoy, Oğuzhan \& Zekeriya, Erkin. "TRADE: A Transparent, Decentralized Traceability System for the Supply Chain". (2018)

[3] Sylim, Patrick \& Liu, Fang \& Marcelo, Alvin \& Fontelo, Paul. "Blockchain technology for detecting falsified and substandard 
drugs in the pharmaceuticals distribution system". In: JMIR Research Protocols. Vol. 7. (2018).

[4] Feng Tian. "An agri-food supply chain traceability system for China based on RFID \& blockchain technology". In: Service Systems and Service Management (ICSSSM), 13th International Conference on. IEEE, pp. 1-6, (2016).

[5] Davis, R. \& Geiger, Bradley \& Gutierrez, Alfonso \& Heaser, Julie \& Veeramani, Dharmaraj. "Tracking blood products in blood centers using radio frequency identification: A comprehensive assessment". In: Vox Sanguinis, vol. 91, pp. 50-60, (2009).
[6] A. Jabbarzadeh, F. Behnam and S. Stefan. "Dynamic supply chain network design for the supply of blood in disasters: A robust mode with real-world application". In: Transportation Research Part E: Logistics and Transportation Review, vol. 70, pp. 225-244, (2014).

[7] Satoshi Nakamoto. "Bitcoin: A peer-to-peer electronic cash system". (2008).

[8] Saveen A Abeyratne and Radmehr P Monfared. "Blockchain ready manufacturing supply chain using distributed ledger". (2016).

[9] Times of India, Article on Blood Wastage in Maharashtra. (2017). 